\title{
Nanostructure of Si-Ge Near-Surface Layers Produced by Ion Implantation and Laser Annealing
}

\author{
D. Klinger, S. Kret, J. Auleytner and D. ŻYmierskA* \\ Institute of Physics, Polish Academy of Sciences \\ al. Lotników 32/46, 02-668 Warsaw, Poland
}

\begin{abstract}
An annealing with the nanosecond laser light pulse is applied for crystal lattice reconstruction of a disturbed near-surface layer, which was created in semiconductor material as a result of the implantation process. Radiation with energy density higher than the threshold value causes the melting of the surface layer and then the epitaxial recrystallization from the melt on a different substrate. Structural changes occurring in the Ge implanted Si crystals after annealing with different energy densities are investigated by means of the cross-section high-resolution transmission electron microscopy.
\end{abstract}

PACS numbers: 61.72.Cc, 61.72.Ff, 61.72.Ji, 61.72.Nn, 61.80.Ba

\section{Introduction}

As a result of ion implantation with high dose a surface layer with very disordered structure is created. For lattice reconstruction, among other methods, the laser pulse annealing (LPA) can be used. The structure quality of recovered layer is dependent on the energy density of the laser beam falling on target surface. The pulse laser annealing with the high enough value of the energy density causes the epitaxial recrystallization from the melt on a solid-state matrix [1, 2].

In our previous papers we have reported theoretical and experimental investigations of defect structure induced by the pulsed laser annealing with different energy densities in Ge implanted silicon crystal [3, 4]. Numerical calculations of transient temperature profiles in the irradiated target were performed to determine the dependence of the melt depth on the radiation energy density. The optimal value was defined as an energy density of one laser pulse causing the melting of

*corresponding author; e-mail: zymier@ifpan.edu.pl 
the whole disturbed near-surface layer and then the epitaxial recrystallization from the melt on a defect-free crystal matrix. Changes caused by the LPA in the structure of the near-surface layer were investigated by means of such complementary methods as Rutherford backscattering spectroscopy (RBS), X-ray section topography and optical observations with the Nomarski microscope. It has been shown that exceeding the optimal value of radiation energy density can create the misfit dislocations of large density.

The presented paper reports in detail the defect structure induced by the scanned laser annealing of the pulsed nanosecond radiation with different energy densities. To study the annealed areas we applied the high-resolution transmission electron microscopy (HRTEM) technique.

\section{Experiment}

The dislocation free Czochralski grown Si (Cz-Si) crystal of the [111] orientation was chemo-mechanically polished on both sides and then it was implanted at the room temperature with $80 \mathrm{keV}$ Ge ions at a dose of $5 \times 10^{15} / \mathrm{cm}^{2}$. As a result of the implantation process a fully amorphized near-surface layer was formed. The amorphization was confirmed by the reflection high-energy electron diffraction (RHEED) method. Next, the sample was annealed by the nanosecond laser pulse radiation. The UV beam of $308 \mathrm{~nm}$ wavelength was generated by the Lambda Physik LPX100 XeCl excimer laser. The Rutherford channeling measurements using the $2 \mathrm{MeV} H e$ ion beam were performed to determine the amorphous layer thickness and to compare the structure of the non-implanted area with the structure of the implanted and annealed ones. The morphology of the sample surface was investigated by means of the interference-polarizing Nomarski microscope. Details of the irradiation procedure and results of the investigations were reported in the previously published papers [3, 4].

The electron microscopy observations were performed at the University of Cannes (France) on the Philips CM 20 UT microscope, operating at accelerating voltage of $200 \mathrm{kV}$. The specimens for electron microscopy observations were prepared in cross-section by mechanical polishing and argon ion milling. The defect structure was studied by the high-resolution transmission electron microscopy using both bright field and 9-beam technique.

\section{Results}

The ion implantation caused the creation of a thin near-surface layer with disordered structure lying on the crystalline matrix, and the formation of a layer with enlarged concentration interstitials located at the amorphous-crystal interface $[5,6]$. From the analysis of the RBS measurements it can be concluded that the structure of the near-surface region was disturbed up to depth of about $135 \mathrm{~nm}$. In the area irradiated with the energy density near the calculated optimal value 
$\left(\rho_{1}=0.6 \mathrm{~J} / \mathrm{cm}^{2}\right)$ almost fully disturbed layer was melted and then recrystallized on the crystalline Si matrix. The RBS measurements obtained for this area testify to the recovery of the near-surface layer structure in about $70 \%$. Figure 1 shows a high resolution TEM image of this annealed region. We can observe a well



Fig. 1. (111) HRTEM image shows a quasi-perfect crystal structure after annealing with the irradiation energy density near the calculated optimal value $\left(\rho_{1}=0.6 \mathrm{~J} / \mathrm{cm}^{2}\right)$. Very small isolated circular regions (marked by arrows) are visible.
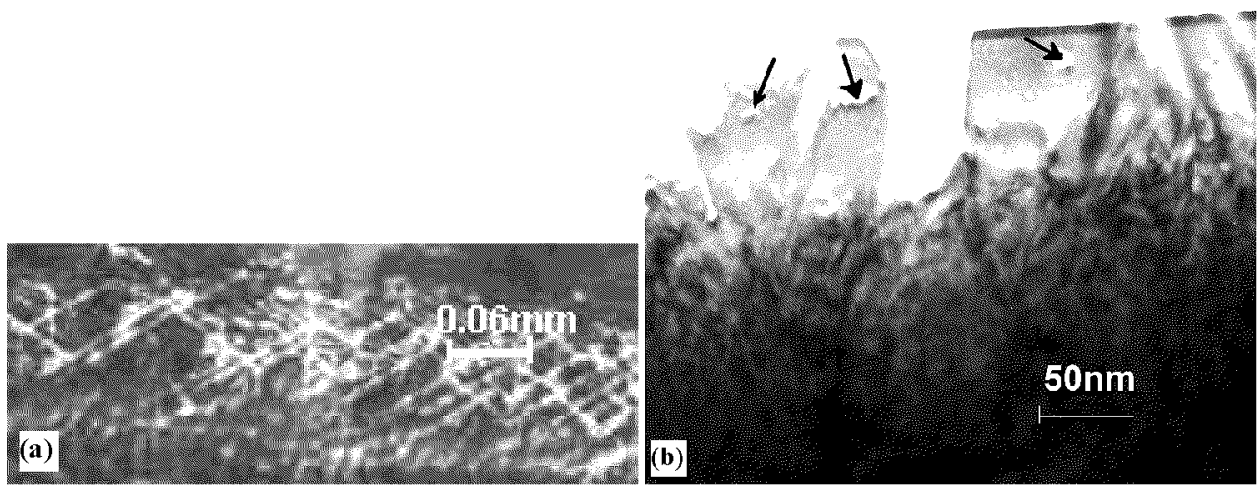

Fig. 2. Images obtained for the area annealed with the beam energy density considerably exceeding the optimal value $\left(\rho_{2}=3 \mathrm{~J} / \mathrm{cm}^{2}\right)$. (a) Surface with misfit dislocations of large density taken from the Nomarski microscope. (b) (111) HRTEM image with large columnar grains creating a quasi-texture and amorphous regions among them. At the interface recrystallized layer-crystal matrix a damage structure is visible. In the interior of the columns the extended defects like bubbles (marked by arrows) can be observed. 
visible picture of lattice planes and very small isolated circular regions (marked by arrows) with different contrast in relation to the image of a perfect structure. The explanation of the nature of these objects is difficult. We suppose that they were created as a consequence of the oxygen atoms building in the inner Si structure or of formation of Ge quantum dots $[7,8]$.

In the area annealed with the beam energy density considerably exceeding the optimal value $\left(\rho_{2}=3 \mathrm{~J} / \mathrm{cm}^{2}\right)$ not only the disturbed near-surface layer but also the layer of the crystalline matrix were melted. Figure 2 a shows the picture of this region obtained from the Nomarski microscope. On the surface we observed the dislocation network, which testifies to creating the epitaxial crystal layer with the misfit dislocations of large density. The high-resolution image obtained for
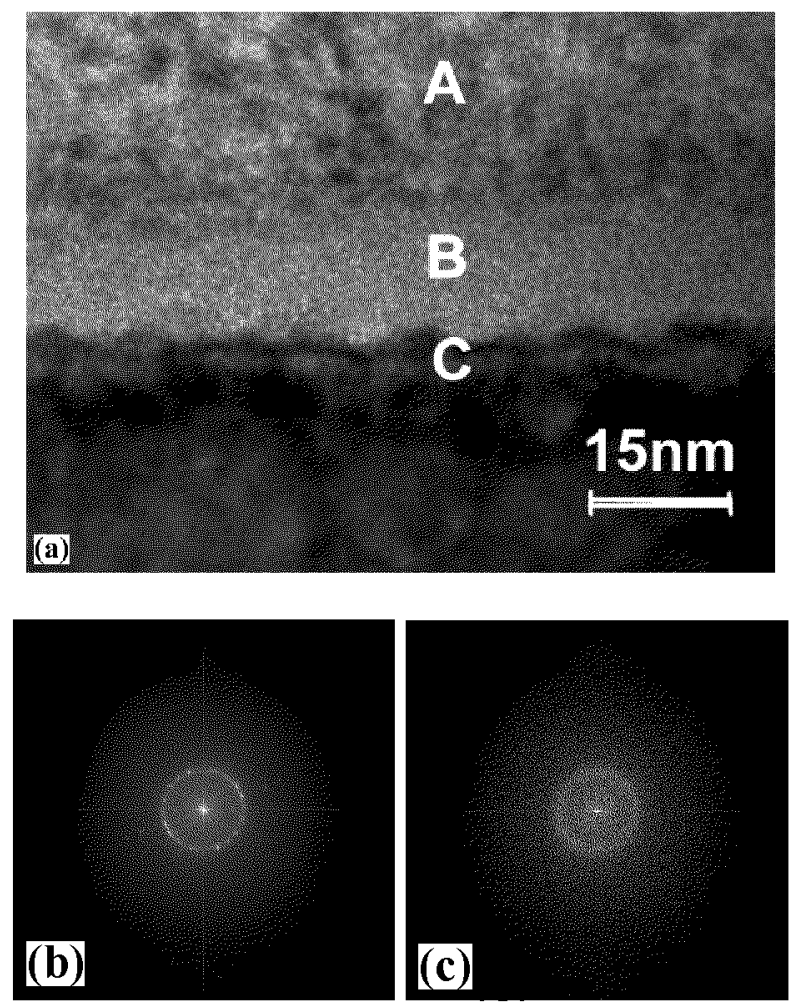

Fig. 3. HRTEM images obtained for the area annealed with the energy density lower than the calculated optimal value $\left(\rho_{3}=0.45 \mathrm{~J} / \mathrm{cm}^{2}\right)$. (a) Cross-section image: A - the part of the disturbed layer melted and epitaxially recrystallized, B - the amorphized layer created as a result of the ion implantation, not melted, $\mathrm{C}-$ the layer with disordered structure at the interface amorphized layer-crystal matrix; (b) diffraction pattern corresponding to the polycrystalline layer A; (c) diffraction pattern corresponding to the previously amorphized and not melted layer B. 
this annealed region is presented in Fig. 2b. The large columnar grains creating a quasi-texture and growing from the substrate to the surface with a lateral dimension of about $100 \mathrm{~nm}$ were formed in the consequence of the recrystallization from the melt. Between the columns the amorphous regions are visible. In the interior of the columns we can observe extended defects like bubbles (marked by arrows). The existence of the damage structure in the crystalline matrix is visible at the interface recrystallized layer — defect-free crystal matrix.

Figure $3 \mathrm{a}$ shows the high-resolution image obtained for the area annealed with the energy density lower than the calculated optimal value $\left(\rho_{3}=0.45 \mathrm{~J} / \mathrm{cm}^{2}\right)$. In this case the annealing caused less pronounced changes in the structure of the near-surface layer. Only the part of the disturbed layer (marked by A) was melted and then it was epitaxially recrystallized on the amorphous substrate (marked by B). At the interface amorphized layer - crystal matrix the layer with disordered structure (marked by C), created as a result of the implantation process is visible. In Fig. $3 \mathrm{~b}$ and $\mathrm{c}$ the diffraction patterns corresponding to the polycrystalline layer (A) and to the part of previously amorphized and not melted layer (B) are shown.

\section{Conclusions}

The high-resolution transmission electron microscopy images showed many details of the defect structure induced by the scanned laser annealing of the pulsed nanosecond radiation with different energy densities.

In quasi-perfect crystal structure obtained in the case of annealing with the energy density near the calculated optimal value we observed local isolated circular regions created as a consequence of the oxygen atoms building in the inner $\mathrm{Si}$ structure or of the formation of Ge quantum dots.

On the surface of the area annealed with the beam energy density considerably exceeding the optimal value, the misfit dislocations of a large density are formed. In this region a quasi-texture with extended defects like bubbles were observed.

Annealing with the energy density lower than the calculated optimal value leads to creation of the epitaxial polycrystalline layer on the amorphous substrate.

Results of all performed investigations showed that such complementary methods as HRTEM, RBS, RHEED, X-ray section topography and optical observations by means of the Nomarski microscope are necessary for full characterization of the structural changes caused by the LPA.

\section{Acknowledgments}

The authors are grateful to Prof. B. Kozankiewicz of the Institute of Physics of the Polish Academy of Sciences for help in the LPA experiment, to Prof. A. Turos of the Institute of Nuclear Studies for cooperation in the RBS measurements, 
as well as to Dr. P. Dłużewski of the Institute of Physics of PAS for discussion concerning the interpretation of the HRTEM images.

This study was partly supported by the State Committee for Scientific Research under grant No. 2P03B 09516.

\section{References}

[1] M. Berti, G. Mazzi, L. Calcagnile, A.V. Drigo, P.G. Merli, A. Migliori, J. Mater. Res. 6, 2120 (1991).

[2] L. Calcagnile, Phys. Status Solidi A 151, 23 (1995).

[3] D. Klinger, M. Lefeld-Sosnowska, D. Żymierska, J. Auleytner, B. Kozankiewicz, K. Regiński, Phys. Status Solidi A 171, 389 (1999).

[4] D. Klinger, M. Lefeld-Sosnowska, J. Auleytner, D. Żymierska, L. Nowicki, A. Stonert, S. Kwiatkowski, J. Alloys Comp. 328, 242 (2001).

[5] D.K. Sadana, W. Maszara, J.J. Wortmann, G.A. Rozgonyi, W.K. Chu, J. Electrochem. Soc. 131, 943 (1984).

[6] J. Morawiec, Acta Phys. Pol. A 85, 819 (1994).

[7] A. Sakai, T. Tatsumi, Appl. Phys. Lett. 64, 52 (1994).

[8] O. Leifeld, R. Hartmann, E. Muller, E. Kaxiras, K. Kern, D. Grutzmacher, Nanotechnology 10, 122 (1999). 